Discovery of Radio Emission From Transient Anomalous X-Ray Pulsar XTE J1810-197

J. P. Halpern, E. V. Gotthelf, R. H. Becker, D. J. Helfand, R. L. White

November 11, 2005

The Astrophysical Journal Letters 
This document was prepared as an account of work sponsored by an agency of the United States Government. Neither the United States Government nor the University of California nor any of their employees, makes any warranty, express or implied, or assumes any legal liability or responsibility for the accuracy, completeness, or usefulness of any information, apparatus, product, or process disclosed, or represents that its use would not infringe privately owned rights. Reference herein to any specific commercial product, process, or service by trade name, trademark, manufacturer, or otherwise, does not necessarily constitute or imply its endorsement, recommendation, or favoring by the United States Government or the University of California. The views and opinions of authors expressed herein do not necessarily state or reflect those of the United States Government or the University of California, and shall not be used for advertising or product endorsement purposes. 
To appear in The Astrophysical Journal Letters

\title{
Discovery of Radio Emission from Transient Anomalous X-ray Pulsar XTE J1810-197
}

\author{
J. P. Halpern ${ }^{1}$, E. V. Gotthelf ${ }^{2}$, R. H. Becker ${ }^{3}$, D. J. Helfand ${ }^{1}$, and R. L. White ${ }^{4}$
}

\begin{abstract}
We report the first detection of radio emission from any anomalous X-ray pulsar (AXP). Data from the Very Large Array (VLA) MAGPIS survey with angular resolution $6^{\prime \prime}$ reveals a point-source of flux density $4.5 \pm 0.5 \mathrm{mJy}$ at 1.4 $\mathrm{GHz}$ at the precise location of the 5.54 s pulsar XTE J1810-197. This is greater than upper limits from all other AXPs and from quiescent states of soft gammaray repeaters (SGRs). The detection was made in 2004 January, 1 year after the discovery of XTE J1810-197 during its only known outburst. Additional VLA observations both before and after the outburst yield only upper limits that are comparable to or larger than the single detection, neither supporting nor ruling out a decaying radio afterglow related to the X-ray turn-on. Another hypothesis is that, unlike the other AXPs and SGRs, XTE J1810-197 may power a radio synchrotron nebula by the interaction of its particle wind with a moderately dense environment that was not evacuated by previous activity from this least luminous, in X-rays, of the known magnetars.
\end{abstract}

Subject headings: pulsars: general — pulsars: individual (XTE J1810-197, AX J1844.8-0256) — radio continuum: stars - X-rays: stars

\footnotetext{
${ }^{1}$ Department of Astronomy, Columbia University, 550 West 120th Street, New York, NY 10027; jules,djh@astro.columbia.edu

${ }^{2}$ Columbia Astrophysics Laboratory, Columbia University, 550 West 120th Street, New York, NY 10027; eric@astro.columbia.edu

${ }^{3}$ Physics Department, University of California, 1 Shields Avenue, Davis, CA 95616; and Institute of Geophysics and Planetary Physics, Lawrence Livermore National Laboratory, Livermore, CA 94550; bob@igpp.ucllnl.org

${ }^{4}$ Space Telescope Science Institute, 3700 San Martin Drive, Baltimore, MD 21218; rlw@stsci.edu
} 


\section{Introduction}

According to the magnetar theory of AXPs and SGRs (Duncan \& Thompson 1992), the high-energy radiation of these slow pulsars $(5<P<12 \mathrm{~s})$ is supplied not by magnetic braking of their rotation, which is insufficient, but from particle acceleration and/or internal heating that is powered by the decay of an enormous magnetic field $\left(B \geq B_{\mathrm{QED}}=4.4 \times\right.$ $10^{13} \mathrm{G}$ ). The absence of radio emission is a defining characteristic of AXPs. Radio emission from magnetars has only been seen following impulsive high-energy events from two SGRs. Specifically, a radio source detected within a few days of the intense 1998 August $27 \gamma$-ray burst from SGR 1900+14 faded rapidly during the following month (Frail, Kulkarni, \& Bloom 1999). The giant flare of SGR 1806-20 on 2004 December 27 (Hurley et al. 2005; Palmer et al. 2005) was accompanied by a radio source that exhibited a complex decay (Cameron et al. 2005; Gaensler et al. 2005b). No radio transient has been recorded from an AXP, e.g., after a bursting episode of 1E 2259+586 (Kaspi et al. 2002), and no persistent radio emission, whether compact or extended, has been clearly attributed to an AXP or an SGR.

The transient AXP XTE J1810-197 is a $5.54 \mathrm{~s}$ X-ray pulsar discovered by Ibrahim et al. (2004) using the Rossi X-ray Timing Explorer (RXTE), and located to subarcsecond precision by Chandra and infrared counterpart detections (Gotthelf et al. 2004; Israel et al. 2004). When discovered in a high state, the source was already decaying exponentially with a time constant of 270 days (Ibrahim et al. 2004) from a recorded maximum flux $F_{X}(2-10 \mathrm{keV}) \approx 6 \times 10^{-11} \mathrm{ergs}^{-2} \mathrm{~s}^{-1}$. The onset and initial properties of the outburst were not observed, but $R X T E$ scans restrict the epoch of the event to between 2002 November and 2003 January. Archival X-ray detections of the previously anonymous source indicate a steady quiescent state with flux two orders-of-magnitude less than the maximum measured

value. Detailed interpretation of the decaying X-ray outburst of XTE J1810-197 using $X M M-N e w t o n$, as well as its historical X-ray properties, were reported by Gotthelf et al. (2004), Halpern \& Gotthelf (2005), and Gotthelf \& Halpern (2005).

This Letter presents a unique VLA detection of XTE J1810-197 1 year after its X-ray outburst, and upper limits from other archival VLA data that restrict its spectrum and pattern of variability. Possible origins of the radio emission are explored, and comparisons are made to upper limits from other AXPs, and to transient radio detections that followed outbursts of SGRs. Although the distance to XTE J1810-197 is highly uncertain, we adopt here $d=2.5 \mathrm{kpc}$ following the argument in Gotthelf \& Halpern (2005). This is a revision of the larger estimates used in earlier papers. 


\section{Observations and Analysis}

Observations at $1.4 \mathrm{GHz}$ of the region containing XTE J1810-197 were obtained on several days in 2004 January as part of the VLA Multi-Array Galactic Plane Imaging Survey (MAGPIS; Helfand, Becker, \& White 2005)1 A total of 15 min of exposure were accumulated in B configuration, with a resulting beam FWHM $=6^{\prime \prime}$ and rms sensitivity $0.5 \mathrm{mJy}$ per beam. Figure 1 shows the resulting image centered on the Chandra coordinates of XTE J1810-197 given in Gotthelf et al. (2004), R.A. $18^{\mathrm{h}} 09^{\mathrm{m}} 51.08$, Decl. - $19^{\circ} 43^{\prime} 51^{\prime \prime} 7$ (J2000.0). A definite radio source is detected with flux density $4.5 \pm 0.5$ mJy at coordinates R.A. $18^{\mathrm{h}} 09^{\mathrm{m}} 51^{\mathrm{s}} 14$, Decl. $-19^{\circ} 43^{\prime} 51^{\prime \prime} 2$ (J2000.0), within $1^{\prime \prime}$ of the Chandra position, which in turn is identical to the coordinates of its IR counterpart (Israel et al. 2004). The difference is comparable to the combined astrometric uncertainty of both instruments. Since the radio source is the only one within a $3^{\prime} .5$ radius of the pulsar, the probability that it is a chance coincidence of an unrelated object within a radius of $1^{\prime \prime}$ can be estimated at $\sim 2 \times 10^{-5}$, and the identification on the basis of position alone is compelling. The radio source extent is unresolved, from which we infer an upper limit on its intrinsic radius of $3^{\prime \prime}$, or $r<1.1 \times 10^{17} d_{2.5} \mathrm{~cm}$, where $d_{2.5}$ is the distance in units of $2.5 \mathrm{kpc}$.

We then analyzed several VLA observations from earlier Galactic plane surveys (White, Becker, \& Helfand 2005) and other archival data, with results listed in Table 1. Only upper limits are obtained from these observations, including the ones reported by Gaensler \& Brogan (2003). When quoted at the $3 \sigma$ level, nearly all of the upper limits are higher than the single detection. Therefore, they contain little information about intrinsic variability of the source. However, two VLA upper limits obtained in 2003, following the X-ray outburst of XTE J1810-197, restrict an hypothesized power-law decay to $t^{-0.6}$ or slower. The upper limit of $3.6 \mathrm{mJy}$ in 2004 March is then in mild conflict with the detection in 2004 January. If, on the other hand, the source is truly constant, then the spectrum from $0.33-4.8 \mathrm{GHz}$ cannot be fitted with a single power law of the form $F_{\nu} \propto \nu^{\alpha}$. The upper limit of $1 \mathrm{mJy}$ at $4.8 \mathrm{GHz}$ requires $\alpha<-1.1$ between 1.4 and $4.8 \mathrm{GHz}$, while the upper limit of $10.5 \mathrm{mJy}$ at $330 \mathrm{MHz}$ requires $\alpha>-0.66$ between $330 \mathrm{MHz}$ and $1.4 \mathrm{GHz}$. The implied spectral curvature might be intrinsic, or the onset of self absorption; alternatively, variability is required.

We also inspected the MAGPIS $1.4 \mathrm{GHz}$ B configuration image for another transient AXP candidate, AX J1844.8-0256 (Gotthelf \& Vasisht 1998; Torii et al. 1998), which yields a $3 \sigma$ upper limit of $0.5 \mathrm{mJy}$ for a point source at R.A. $18^{\mathrm{h}} 44^{\mathrm{m}} 54.69$, Decl. $-02^{\circ} 56^{\prime} 53$. $^{\prime \prime} 4$ (J2000.0). We derived this position from the sum of several archival Chandra images. This 6.97 s pulsar associated with a supernova remnant (Gaensler, Gotthelf, \& Vasisht 1999)

\footnotetext{
${ }^{1}$ http://third.ucllnl.org/gps
} 
remains only a candidate AXP because its period derivative has not yet been measured due to a dramatic reduction in its X-ray flux (Vasisht et al. 2000).

\section{Discussion}

No radio pulsations have been detected from AXPs, with pulsed flux density limits $<0.1 \mathrm{mJy}$ at $1.4 \mathrm{GHz}$ obtained for most sources (Mereghetti et al. 2002). It is not yet established by statistics alone whether the small set of AXPs are intrinsically radio quiet or just unfavorably oriented, their long periods implying small active polar caps and narrow beams. Neither has persistent radio emission been seen from this class until now, although flux limits from interferometric imaging, listed in Table 2, are generally not as deep as the singledish searches for pulsed flux. Although the VLA source associated with XTE J1810-197 is spatially unresolved, the bulk of its flux is unlikely to be due to magnetospheric pulsar emission. A $4.5 \mathrm{mJy}$ source pulsed at the $5.54 \mathrm{~s}$ X-ray period should be easily detected in the Parkes Multibeam Pulsar Survey operating at $1.4 \mathrm{GHz}$, as have other long-period, high $B$-field pulsars (e.g., McLaughlin et al. 2003) that are at least an order-of-magnitude fainter. Therefore, we assume that most of the emission is incoherent synchrotron radiation arising from well outside the light cylinder, $r_{\mathrm{lc}}=2.6 \times 10^{10} \mathrm{~cm}$, but within the VLA resolution limit $r=10^{17} \mathrm{~cm}$. Its brightness temperature is then $T_{\mathrm{B}}=100\left(d_{2.5} / r_{17}\right)^{2} \mathrm{~K}$, and it is probably larger than $10^{12} \mathrm{~cm}$, assuming that $T_{\mathrm{B}}<10^{12} \mathrm{~K}$.

To put the unique radio detection of XTE J1810-197 in context, we note that its radio flux density is larger than all of the upper limits from other AXPs (Table 2), but its radio luminosity is demonstrably larger than only two or three, considering that the distances to AXPs, as tabulated by Mereghetti et al. (2002) and Woods \& Thompson (2006), may be uncertain by a factor of 2 . The radio detection of XTE J1810-197 may be a significant clue into the physics of its class. XTE J1810-197 differs from other AXPs in two respects. First, it is transient, with an X-ray luminosity that is comparable to the persistent AXPs only near the peak of its outburst. Second, its quiescent state may be long-lived, so that its time-averaged X-ray luminosity could be two orders-of-magnitude less than the others. The only other transient X-ray source in Table 2 is AX J1844.8-0256, but its distance is uncertain and possibly large (Gaensler et al. 1999), so its radio luminosity is not necessarily smaller than that of XTE J1810-197.

An obvious hypothesis, that the 2004 January radio luminosity of XTE J1810-197 is a transient afterglow that originated with the 2003 January X-ray outburst, is problematic. First, there is the twice documented absence of a more luminous radio source in the months following the X-ray outburst, in 2003 February and April. It is also notable that no radio 
source, to a limit of $50 \mu \mathrm{Jy}$, was detected 2 days after an X-ray outburst and glitch of the AXP 1E 2259+586 (Kaspi et al. 2002). Second, there was no evidence for any soft $\gamma$-ray outburst from XTE J1810-197 resembling the flares of the SGRs, only a slowly decaying, thermal X-ray spectrum that integrated over a time scale of 300 days to a total energy of $\sim 4 \times 10^{42} d_{2.5}^{2} \operatorname{ergs}$ (Gotthelf \& Halpern 2005). In fact, it is possible that XTE J1810-197 was never more luminous than a normal AXP. Its activity is perhaps better characterized as a turn-on than an outburst, and is in marked contrast to the $10^{46}$ ergs $\gamma$-ray flare of SGR 1806-20 in 2004 December (Hurley et al. 2005; Palmer et al. 2005).

Third, only in the aftermath of giant flares from the SGR 1806-20 and 1900+14 have decaying radio sources been detected by the VLA, and even these were faint and short-lived. In the case of SGR 1900+14, the peak $1.4 \mathrm{GHz}$ flux density 10 days after the 1998 August flare was 0.7 mJy (Frail et al. 1999), but it decayed as a power law of index -2.6 and was no longer detectable after 1 month. Just 1.6 days after another burst activation from SGR $1900+14$ in 2001 April, no radio emission was detected to a $5 \sigma$ limit of $0.45 \mathrm{mJy}$ at $5 \mathrm{GHz}$ (Kouveliotou et al. 2001), which was not unexpected since the $\gamma$-ray fluence of the 2001 burst was 25 times smaller than the 1998 burst. The 2004 December giant flare from SGR 1806-20 produced a radio source initially detected at $178 \mathrm{mJy}$ at $1.4 \mathrm{GHz}$, which faded to 10 mJy after 50 days (Cameron et al. 2005). Extrapolated to 1 year, SGR 1806-20 should be as faint in the radio as XTE J1810-197 was 1 year after its X-ray turn-on. This despite the fact that the $\gamma$-ray flare of SGR 1806-20 was three orders-of-magnitude more luminous than the entire extrapolated X-ray luminosity of XTE J1810-197 from the peak of its turn-on to the present time.

For the sake of completeness, we note that at least the kinematic properties of the SGR events are not incompatible with the size of the XTE J1810-197 radio source. If we assume that the radio emitting plasma observed in 2004 January was first ejected from the neutron star a year earlier, then we can place an upper limit on the ejection velocity, $v / c<0.12 d_{2.5}$. This is comparable to the velocity directly estimated in the case of SGR 1806-20 from its resolved VLA images, $v / c=(0.27 \pm 0.10) d_{15}$ (Gaensler et al. 2005b).

If the radio emission from XTE J1810-197 is not a transient afterglow, it may be caused by a variable wind of high-energy particles, which we hypothesize could be responsible for powering a small synchrotron emitting nebula under favorable circumstances. The spin-down rates of AXPs fluctuate by a large factor, which is taken as evidence that rotational energy is regularly removed by mechanisms other than dipole braking, e.g., a particle wind (Thompson \& Blaes 1998; Harding, Contopoulos, \& Kazanas 1999). Continued monitoring by RXTE and XMM-Newton shows that the spin-down rate of XTE J1810-197 varies irregularly by at least a factor of 4 , with $\dot{P}=(0.5-2.2) \times 10^{-11} \mathrm{~s} \mathrm{~s}^{-1}$ (Ibrahim et al. 2004; Gotthelf et 
al. 2004; Gotthelf \& Halpern 2005). Assuming a moment of inertia $I=10^{45} \mathrm{~g} \mathrm{~cm}^{2}$, this implies the possibility of a wind of luminosity $\dot{E}=4 \pi^{2} I \dot{P} / P^{3} \sim 4 \times 10^{33} \mathrm{ergs} \mathrm{s}^{-1}$, which is comparable to the quiescent thermal X-ray luminosity (Halpern \& Gotthelf 2005), and five orders-of-magnitude larger than the radio luminosity $\nu L_{\nu} \approx 5 \times 10^{28} \mathrm{ergs} \mathrm{s}^{-1}$ at $1.4 \mathrm{GHz}$. In addition, $R X T E$ continued to find small X-ray bursts from XTE J1810-197 long after its initial outburst. Woods et al. (2005) document four such bursts in 2003 September, 2004 February, and 2004 May, from a total exposure time of 9 days. Although these have thermal spectra and blackbody radii much smaller than the surface area of a neutron star, their temperatures of 4-8 keV imply local flux greater than the Eddington value. Whether their initial trigger is magnetic reconnection or crust fracturing, they likely cause matter to be ejected from the surface. The strongest of these bursts had a energy of $3.5 \times 10^{37} d_{2.5}^{2} \operatorname{ergs}$, and together they emitted $4.2 \times 10^{37} d_{2.5}^{2}$ ergs. Therefore we can estimate a time-averaged luminosity in bursts of $\dot{E} \sim 5.4 \times 10^{31} \mathrm{ergs} \mathrm{s}^{-1}$, which is two orders-of-magnitude less than the estimated wind luminosity.

It is possible that long-lived radio emission is powered by the accumulated plasma ejected in the process of creating the X-ray bursts, as well as by the more luminous wind. Following the arguments of Frail \& Scharringhausen (1997) and Gaensler et al. (2000), the conversion of the ubiquitous pulsar winds to an observable pulsar wind nebula (PWN) may be limited not by the energy carried in the wind, but by the environment - specifically, by whether the ambient pressure is large enough to shock the wind and confine the nebula. In the case of XTE J1810-197, balancing the assumed wind power $\dot{E} \sim 4 \times 10^{33} \mathrm{ergs} \mathrm{s}^{-1}$ at a termination shock radius $r_{\mathrm{w}}<10^{17} \mathrm{~cm}$ requires a pressure $\dot{E} / 4 \pi r_{\mathrm{w}}^{2} c=1.1 \times 10^{-12}\left(r_{\mathrm{w}} / 10^{17}\right)^{-2}$ dynes cm $^{-2}$. This is a rather modest value even if the true radius of the nebula is only $10^{16} \mathrm{~cm}$ and the pressure is $1 \times 10^{-10}$ dynes $\mathrm{cm}^{-2}$, or alternatively, if the wind power has been 100 times larger than assumed here. In comparison, Helfand, Gotthelf, \& Halpern (2001) showed that the radius of the Vela PWN is consistent with the thermal pressure in the surrounding hot supernova remnant, $8.5 \times 10^{-10}$ dynes $\mathrm{cm}^{-2}$ measured from X-ray spectroscopy (Markwardt \& Ögelman 1997). There is no evidence for a supernova remnant surrounding XTE J1810-197, which may imply that the surrounding temperature is cooler. If we assume an ionized medium of $T=10^{4} \mathrm{~K}$, then the required hydrogen density is $n_{\mathrm{H}} \sim 0.4\left(r_{\mathrm{w}} / 10^{17}\right)^{-2}\left(T / 10^{4}\right)^{-1}$ $\mathrm{cm}^{-3}$. The actual development of a PWN is a time-dependent process, with a forward shock radius estimated as $r_{\mathrm{s}}(t)=\left(\dot{E} / 4 \pi \rho_{0}\right)^{1 / 5} t^{3 / 5}$ (Arons 1983). For the numerical values assumed here, $r_{\mathrm{s}} \leq 10^{17} \mathrm{~cm}$ is reached in $\leq 100$ years. Alternatively, if the pulsar's space velocity $v_{\mathrm{p}}$ exceeds $\dot{r}_{\mathrm{s}}$, then a bow-shock nebula will form with apex radius $r_{\mathrm{a}}=\left(\dot{E} / 4 \pi \rho_{0} v_{\mathrm{p}}^{2}\right)^{1 / 2}$ that is smaller than $r_{\mathrm{s}}$ for a "static" nebula.

We note that the radio emitting efficiency of XTE J1810-197, as defined by Frail \& Scharringhausen (1997) and Gaensler et al. (2000), is comparable to the upper limits 
obtained by them for rotation-powered pulsars of similar $\dot{E}$ as we are assuming for the wind of XTE J1810-197. Therefore, evidence is lacking that the average wind power of XTE J1810-197 is much greater than its spin-down value. Additionally, there is no X-ray PWN around XTE J1810-197, whose image is consistent with a point-source at the Chandra resolution of $\mathrm{FWHM} \approx 0^{\prime \prime} .6$ (Israel et al. 2004). Only pulsars with $\dot{E}>10^{36} \mathrm{ergs} \mathrm{s}^{-1}$ have X-ray PWNe (Gotthelf 2004). Perhaps the difference between XTE J1810-197 and other magnetars is that XTE J1810-197 is surrounded by a dense medium, while the past activity of the other, more energetic magnetars, has disrupted and evacuated their surroundings. We note that this is different from the hypothesis advanced by Gaensler et al. (2005a) to explain an apparent H I cavity around AXP 1E 1048.1-5937, which they supposed was blown by the wind of its massive progenitor star.

\section{Conclusions and Future Work}

The detection of radio emission from an AXP comes as a surprise because it is contrary to a defining property of its class. But the physical basis of the classification of XTE J1810-197 is unchanged. The long period, unsteady spin-down, X-ray luminosity exceeding its spindown power, and detailed properties of its two-component X-ray spectrum, are all squarely in the AXP mold, and support the magnetar interpretation (Gotthelf \& Halpern 2005). Although the radio luminosity of XTE J1810-197 is energetically insignificant, a more detailed investigation of its origin could lead to a better understanding of the evolution of AXPs and their immediate surroundings.

The spectral and spatial structure of this compact radio source can be studied with multifrequency observations in the VLA A configuration, which has the potential to resolve the source if it is a PWN powered by a wind of comparable luminosity to the X-ray luminosity or the spin-down power. If size and spectral information are obtained, the particle and magnetic field energies of this presumed synchrotron source can be determined. While the evidence for temporal variability is marginal, variability would not be unexpected, as the Xray source is still declining from its 2003 January turn-on. A monitoring program can study the relationship, if any, of the radio structure and luminosity to the X-ray history. However, it is possible that the environment and the normal X-ray quiescence of XTE J1810-197, rather than its unusual outburst, is what enables it to support a radio source.

Having suggested that XTE J1810-197 is the prototype of a major subclass of young pulsars that may have been detected but not yet recognized in quiescence (Gotthelf et al. 2004), we now anticipate the prospect of an additional marker, in the form of a compact radio source, that will aid in their discovery. 
This work was supported by NASA ADP grant NNG05GC43G to JPH and EVG. RHB and DJH acknowledge the support of the National Science Foundation under grants AST02-6-309 and AST-02-6-55, respectively.

This work was performed under the auspices of the U.S. Department of Energy by University of California, Lawrence Livermore National Laboratory under contract W-7405-Eng-48. 


\section{REFERENCES}

Arons, J. 1983, Nature, 302, 301

Becker, R. H., White, R. L., Helfand, D. J., \& Zoonematkermani, S. 1994, ApJS, 91, 347

Brogan, C. L., Devine, K. E., Lazio, T. J., Kassim, N. E., Tam, C. R., Brisken, W. F., Dyer, K. K., \& Roberts, M. S. E. 2004, AJ, 127, 355

Cameron, P. B., et al. 2005, Nature, 434, 1112

Coe, M. J., Jones, L. R., \& Lehto, H. 1994, MNRAS, 270, 178

Duncan, R. C., \& Thompson, C. 1992, ApJ, 392, L9

Frail, D. A., Kulkarni, S. R., \& Bloom, J. S. 1999, Nature, 398, 127

Frail, D. A., \& Scharringhausen, B. R. 1997, ApJ, 480, 364

Gaensler, B. M., \& Brogan, C. L. 2003, Astron. Telegram, 207, 1

Gaensler, B. M., Gotthelf, E. V., \& Vasisht, G. 1999, ApJ, 526, L37

Gaensler, B. M., McClure-Griffiths, N. M., Oey, M. S., Haverkorn, M., Dickey, J. M., \& Green, A. J. 2005a, ApJ, 620, L95

Gaensler, B. M., Slane, P. O., Gotthelf, E. V., \& Vasisht, G. 2001, ApJ, 559, 963

Gaensler, B. M., Stappers, W., Frail, D. A., Moffett, D. A., \& Johnston, S. 2000, MNRAS, 318,58

Gaensler, B. M., et al. 2005b, Nature, 434, 1104

Gotthelf, E. V. 2004, in IAU Symp. 218, Young Neutron Stars and Their Environments, ed. F. Camilo \& B. M. Gaensler (San Francisco: ASP), 225

Gotthelf, E. V., \& Halpern, J. P. 2005, ApJ, 632, 000 (astro-ph/0506511)

Gotthelf, E. V., Halpern, J. P., Buxton, M., \& Bailyn, C. 2004, ApJ, 605, 368

Gotthelf, E. V., \& Vasisht, G. 1998, NewA, 3, 293

Halpern, J. P., \& Gotthelf, E. V. 2005, ApJ, 618, 874

Harding, A. K., Contopoulos, I., \& Kazanas, D. 1999, ApJ, 525, L125 
Helfand, D. J., Becker, R. H., \& White, R. L. 2005, in preparation

Helfand, D. J., Gotthelf, E. V., \& Halpern, J. P. 2001, ApJ, 556, 380

Hurley, K., et al. 2005, Nature, 434, 1098

Ibrahim, A. I., et al. 2004, ApJ, 609, L21

Israel, G. L., et al. 2004, ApJ, 603, L97

Kaspi, V. M., Gavriil, F. P., Woods, P. M., Jensen, J. B., Roberts, M. S. E., \& Chakrabarty, D. 2002, ApJ, 588, L93

Kouveliotou, C., Tennant, A., Woods, P. M., Weisskopf, M. C., Hurley, K., Fender, R. P., Garrington, S. T., Patel, S. K., \& Göğüs, E. 2001, ApJ, 558, L47

Kriss, G. A., Becker, R. H., Helfand, D. J., \& Canizares, C. R. 1985, ApJ, 288, 703

Markwardt, C. B., \& Ögelman, H. B. 1997, ApJ, 480, L13

McLaughlin, M. A., et al. 2003, ApJ, 591, L135

Mereghetti, S., Chiarlone, L., Israel, G. L., \& Stella, L. 2002, in Proc. 270th WE-Heraeus Seminar on Neutron Stars, Pulsars, and Supernova Remnants, ed. W. Becker, H. Lesch, \& J. Trümper (MPE Rep. 278; Garching: MPE), 29

Palmer, D. M., et al. 2005, Nature, 434, 1107

Thompson, C., \& Blaes, O. 1998, Phys. Rev. D, 57, 3219

Torii, K., Kinugasa, K., Katayama, K., Tsunemi, H., \& Yamauchi, S. 1998, ApJ, 503, 843

Vasisht, G., Gotthelf, E. V., Torii, K., \& Gaensler, B. M. 2000, ApJ, 542, L49

White, R. L., Becker, R. H., \& Helfand, D. J. 2005, AJ, 130, 586

Woods, P. M., Kouveliotou, C., Gavriil, F. P., Kaspi, V. M., Roberts, M. S. E., Ibrahim, A., Markwardt, C. B., Swank, J. H., \& Finger, M. H. 2005, ApJ, 629, 985

Woods, P. M., \& Thompson, C. 2006, in Compact Stellar X-ray Sources, ed. W. H. G. Lewin \& M. van der Klis (Cambridge: Cambridge Univ. Press), in press (astro-ph/0406133)

Zoonematkermani, S., Helfand, D. J., Becker, R. H., White, R. L., \& Perley, R. A. 1990, ApJS, 74, 181

This preprint was prepared with the AAS IATEX macros v5.2. 
Table 1. VLA Observations of XTE J1810-197

\begin{tabular}{lccccc}
\hline \hline \multicolumn{1}{c}{ Dates } & $\begin{array}{c}\text { Frequency } \\
(\mathrm{GHz})\end{array}$ & $\begin{array}{c}\text { Array } \\
\text { Configuration }\end{array}$ & $\begin{array}{c}\text { Beam FWHM } \\
\left({ }^{\prime \prime}\right)\end{array}$ & $\begin{array}{c}F_{\nu} \\
(\mathrm{mJy})\end{array}$ & References \\
\hline 1983 Dec & 1.42 & $\mathrm{~B}$ & 6 & $<5.5$ & $1,2,3$ \\
1990 Oct & 4.82 & $\mathrm{CnB}$ & 6 & $<1$ & $1,3,4$ \\
2001 Jan $-2002 \mathrm{Aug}$ & 0.33 & $\mathrm{~A}, \mathrm{~B}, \mathrm{C}, \mathrm{D}$ & 25 & $<10.5$ & 5,6 \\
2003 Feb & 1.42 & $\mathrm{DnC}$ & $62 \times 35$ & $<10.5$ & 5,6 \\
2003 Apr & 1.42 & $\mathrm{D}$ & 45 & $<7$ & 1,7 \\
2004 Jan & 1.42 & $\mathrm{~B}$ & 6 & $4.5 \pm 0.5$ & 1,7 \\
2004 Mar & 1.42 & $\mathrm{C}$ & 15 & $<3.6$ & 1,7 \\
\hline
\end{tabular}

References. - (1) This work; (2) Zoonematkermani et al. 1990; (3) White et al. 2005; (4) Becker et al. 1994; (5) Gaensler \& Brogan 2003; (6) Brogan et al. 2004; (7) Helfand et al. 2005.

Note. - Upper limits quoted here are $3 \sigma$ based on the rms noise per beam. Uncertainty quoted on the detection is $1 \sigma$. 
Table 2. Imaging Observations of AXPs at $1.4 \mathrm{GHz}$

\begin{tabular}{lcccc}
\hline \hline \multicolumn{1}{c}{ Source } & $\begin{array}{c}F_{\nu} \\
(\mathrm{mJy})\end{array}$ & $\begin{array}{c}d \\
(\mathrm{kpc})\end{array}$ & $\begin{array}{c}F_{\nu} d^{2} \\
\left(\mathrm{mJy} \mathrm{kpc}^{2}\right)\end{array}$ & References \\
\hline $4 \mathrm{U} \mathrm{0142+61}$ & $<0.16$ & 3 & $<1.4$ & 1 \\
1E 1048.1-5937 & $\ldots$ & 2.7 & $\ldots$ & 2 \\
1RXS J170849.0-400910 & $<1.8$ & 5 & $<45$ & 1 \\
XTE J1810-197 & $4.5 \pm 0.5$ & 2.5 & 28 & 3 \\
1E 1841-045 & $<0.36$ & 7 & $<18$ & 4 \\
AX J1844.8-0256 & $<0.5$ & $\sim 8$ & $<32$ & 3 \\
1E 2259+586 & $<0.050$ & 3 & $<0.45$ & 5,6 \\
\hline
\end{tabular}

${ }^{a}$ Transient X-ray source.

References. - (1) Gaensler et al. 2001; (2) Gaensler et al. 2005a; (3) This work; (4) Kriss et al. 1985; (5) Coe et al. 1994; (6) Kaspi et al. 2002.

Note. - Upper limits quoted here are for unpulsed emission at the $3 \sigma$ level. 


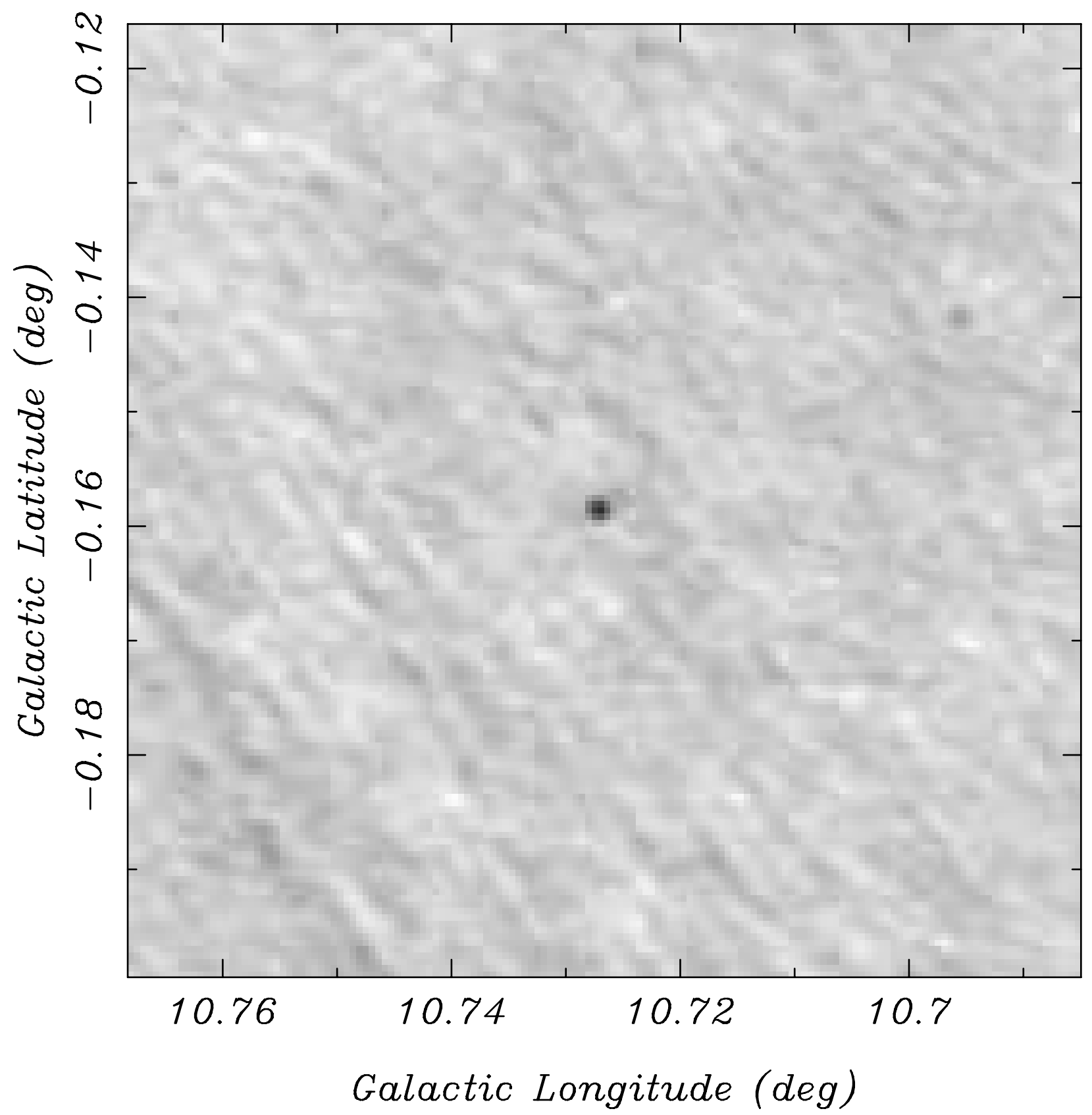

Fig. 1.- MAGPIS VLA image at $1.4 \mathrm{GHz}$ in B configuration of XTE J1810-197 taken in 2004 January. A $9 \sigma$ radio source with flux density $4.5 \pm 0.5 \mathrm{mJy}$ is present within $1^{\prime \prime} .0$ of the X-ray/IR coordinates. This source is unresolved by the $6^{\prime \prime}$ FWHM beam. 